\title{
Collaborative Channel Estimation in Backscattering Tag-to-Tag Networks
}

\author{
Abeer Ahmad \\ Stony Brook University
}

\author{
Akshay Athalye \\ Stony Brook University
}

\author{
Milutin Stanacević \\ Stony Brook University
}

\author{
Samir R. Das \\ Stony Brook University
}

\begin{abstract}
Backscattering Tag-to-Tag Networking (BTTN) represents a rapidly emerging paradigm enabling passive, radio-less tags to communicate directly with each other by reflecting (backscattering) an RF signal supplied by an external un-coordinated exciter. Recent advancements have taken the capability of BTTN beyond basic communication, empowering the networks with the ability to collaboratively sense and recognize human activities in the deployment space. The key to this ability is a novel passive channel estimation, allowing individual tags to measure tag-to-tag wireless channel parameters without involvement of any active radio. Previously reported techniques for this suffer from a limitation in that, they are unable to isolate the tag-to-tag channel of interest from the wider-range exciter-to-tag channels. As a result, the channel estimates and the analytics based thereof are susceptible to dynamic variations and clutter in the overall deployment environment, outside the range of the tag-to-tag link. In this paper, we overcome these limitations using a novel collaborative technique thus greatly enhancing the utility of passive channel estimation in BTTN. We elucidate our proposed technique using analytical modeling and validate with in-lab experiments using tag hardware built from discrete components.
\end{abstract}

\section{INTRODUCTION}

The concept of backscattering which entails wireless communication via reflection of external signals is becoming increasingly popular in a variety of applications [11]. This technology makes for extremely low power transmitters, often called 'tags', which can function without any on-board radios and without externally powered components. In backscattering, the minimal power required for operation is harvested from the external incident signal itself, allowing the tags to be 'passive.' The earliest and most widespread embodiment of this technology, viz. RFID, is primarily used for object identification [2]. However, in the past decade or so, increasing amounts of research efforts on backscattering systems are directed towards enabling diverse forms of wireless communication $[1,3,12]$ including RF-based sensing [4, 9]. This latter capability is of interest in this work.

Most backscattering systems require one end of the communication link to have an 'active' radio component (i.e., 'active' means

Permission to make digital or hard copies of all or part of this work for personal or classroom use is granted without fee provided that copies are not made or distributed for profit or commercial advantage and that copies bear this notice and the full citation on the first page. Copyrights for components of this work owned by others than ACM must be honored. Abstracting with credit is permitted. To copy otherwise, or republish, to post on servers or to redistribute to lists, requires prior specific permission and/or a fee. Request permissions from permissions@acm.org.

DFHS'19, November 10, 2019, New York, NY, USA

(C) 2019 Association for Computing Machinery.

ACM ISBN 978-1-4503-7007-3/19/11 ..\$15.00

https://doi.org/10.1145/3360773.3360882 requiring external power), such as an RFID reader. The latest paradigm emerging in this field is of Backscattering Tag-to-Tag Networking or BTTN which overcomes this limitation allowing passive tags to communicate directly with each other $[6-8,10]$. BTTN does rely on an external source of RF signal that also powers the tag. These 'exciters' could be intentionally deployed, or ambient sources like TV towers or wifi access points, so long as sufficient received power is available [6, 7]. Since the constituent tags are inherently low cost, passive and tiny, one can envision a future where these tags are embedded in the physical infrastructure surrounding our living and working environments and also attached to objects within the environments. This enables a host of 'smart spaces' applications.

Recent advancements have taken the capability of BTTN beyond basic tag-to-tag communication by empowering them with the ability to recognize human activities and interactions in their environment through a unique form of RF sensing [5, 9]. This is achieved without requiring the human subjects to carry any kind of devices (device-free). While the basic idea has been demonstrated in the earlier studies; prior work has significant limitations in terms of isolating and localizing the behavior in a smaller space. The goal of the current paper is on developing the fundamental techniques to overcome this limitation.

\subsection{Limitations of the State of the Art}

A basic setup of a single BTTN link for human activity sensing is shown in Figure 1.

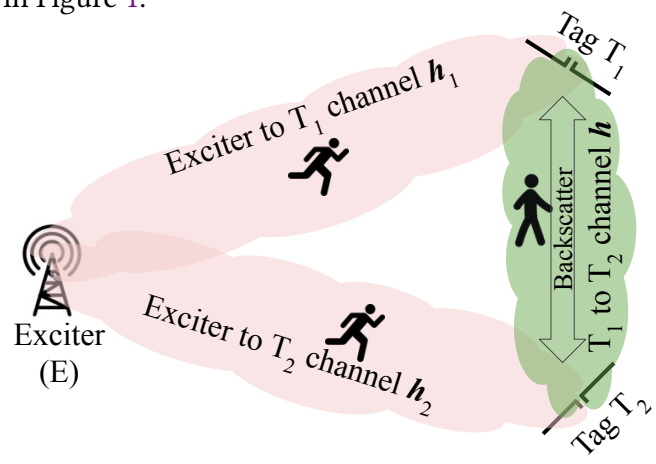

Figure 1: A single passive tag-to-tag link for human sensing

Two tags $T_{1}$ and $T_{2}$ are communicating with each other using backscattering of a signal provided by a single excitation source $E$. BTTN tags perform activity sensing by employing passive channel estimation which measures the channel parameters of a tag-to-tag link [9]. Dynamic patterns in these channel parameters are used to recognize specific activities. Typically, the exciter to tag channels $\mathbf{h}_{1}$ and $\mathbf{h}_{2}$ span large areas while the tag-to-tag channel $\mathbf{h}$ spans a much smaller area in the vicinity of the two communicating tags (Figure 1). Thus for fine-grain activity sensing, it is best if each 
tag-to-tag link can "see" only in its near vicinity and is "blind" to events further away. This, in turn, will allow for multiple users in the environment to be sensed independently and simultaneously by multiple pairs of tags. However, previously reported passive channel estimation techniques are limited in their ability in supporting this scenario $[5,9]$. Specifically, these techniques are unable to eliminate the effects of channels $\mathbf{h}_{1}$ and $\mathbf{h}_{2}$ from the measurement of $\mathbf{h}$. As a result the channel estimates used in performing activity recognition are not purely encapsulating the activity in the vicinity of the link (the green zone), but include dynamic variations and clutter in the entire deployment environment (the red zones). This is detrimental to the desired functionality of human sensing and analytics in BTTN in two ways: (i) It makes the system highly susceptible to environmental clutter and noise. Moreover machine learning (ML) techniques used in activity recognition based on channel patterns will require retraining in different environments. (ii) Simultaneous multi-user activity recognition is extremely challenging since activity in far away regions will interfere with activity within the tag-to-tag channel via the exciter channel.

\subsection{Our Contributions}

In this paper, we focus on passive channel estimation and propose new tag-to-tag channel estimation techniques that do not have the aforementioned limitations. We make the following specific contributions:

(1) We present passive channel estimation techniques that enable the measurement of tag-to-tag channel amplitude and phase decoupled from exciter channel effects.

(2) We implement our proposed technique on tag hardware and demonstrate through experiments that, unlike state-ofthe-art techniques, tag-to-tag channel measurements are unaffected by changes in the exciter channels.

\section{CHANNEL MODELS AND MEASUREMENT TECHNIQUES}

\subsection{Wireless Channels in BTTN}

A single BTTN link as shown in Figure 1 consists of one exciter $E$ and two tags $T_{1}$ and $T_{2}$. The tags in BTTN transmit using backscatter modulation and receive using envelope detection. The general principle of this is similar to prior work in $[6,8]$. The tag-to-tag links are half-duplex in nature, i.e. at any given time, a tag is either in backscattering (or Tx) mode or in receive (or Rx) mode. Backscattering is achieved by changing the impedance connected to the antenna which alters the antenna reflection coefficient $\Gamma$ [8]. This is a complex value with amplitude $|\Gamma|=\rho(0 \leq \rho \leq 1)$ and angle $\angle \Gamma$ $=\phi$. The value $\rho$ determines what fraction of the incident power is backscattered with the phase offset $\phi$. These values are set at the time of tag design [8].

Let us examine the channels when $T_{1}$ is backscattering ( $\mathrm{Tx}$ ) and $T_{2}$ is receiving $(\mathrm{Rx})$. Assume that the exciter emits a continuous wave $(\mathrm{CW})$ signal $A_{E} \cos \left(2 \pi f_{c} t\right)$, where $A_{E}$ is the amplitude of the excitation signal and $f_{c}$ is the carrier frequency. This reaches $T_{1}$ over the multipath channel $\mathbf{h}_{1}$. Let us denote the resultant amplitude (attenuation) and phase of this channel as $a_{h 1}$ and $\theta_{h 1}$ respectively. Then the signal received at $T_{1}$ is $A_{E} \cdot a_{h 1} e^{j \theta_{h 1}}$. Using the above mentioned notations for antenna reflection coefficient, the signal backscattered by $T_{1}$ is $A_{E} \cdot a_{h 1} e^{j \theta_{h 1}} \cdot \rho e^{j \phi}$. This signal gets to $T_{2}$ over the multipath tag-to-tag channel $\mathbf{h}$ with resultant attenuation $a_{h}$ and phase $\theta_{h}$. Simultaneously, $T_{2}$ is also receiving the excitation signal over the multipath $\mathbf{h}_{2}$ channel with resultant attenuation $a_{h 2}$ and phase $\theta_{h 2}$. So the combined received signal at $T_{2}$ is:

$$
\mathrm{S}_{T_{2}}=\underbrace{A_{E}}_{\text {Excitation }}(\underbrace{a_{h 2} e^{j \theta_{h 2}}}_{E \rightarrow T_{2}}+\underbrace{a_{h 1} e^{j \theta_{h 1}}}_{E \rightarrow T_{1}} \cdot \underbrace{\rho e^{j \phi}}_{\text {Tag } \Gamma} \cdot \underbrace{a_{h} e^{j \theta_{h}}}_{T_{1} \rightarrow T_{2}})
$$

Similarly, when $T_{2}$ is backscattering ( $\left.\mathrm{Tx}\right)$ and $T_{1}$ is receiving ( $\left.\mathrm{Rx}\right)$, the combined signal received at $T_{1}$ is:

$$
\mathrm{S}_{T_{1}}=\underbrace{A_{E}}_{\text {Excitation }}(\underbrace{a_{h 1} e^{j \theta_{h 1}}}_{E \rightarrow T_{1}}+\underbrace{a_{h 2} e^{j \theta_{h 2}}}_{E \rightarrow T_{2}} \cdot \underbrace{\rho e^{j \phi}}_{\operatorname{Tag} \Gamma} \cdot \underbrace{a_{h} e^{j \theta_{h}}}_{T_{2} \rightarrow T_{1}})
$$

The passive tag-to-tag channel estimation is achieved by using a technique called multiphase probing (MPP) [8, 9]. Essentially, the Tx tag sends out a short pilot probe repeatedly $k$ times, each time with a different, fixed phase offset $\left(\phi_{k}\right)$. The values and sequence of the phase offsets are $\left(\phi_{k}\right)$ fixed by the design and identical for each tag. On the Rx side, the tag examines the pattern of the received signal amplitude over the various phase slots and formulates estimates of channel parameters.

\subsection{Channel Phase Measurement}

Per the previously reported techniques, the Tx tag sends out the MPP and the Rx tag determines which slot resulted in the maximum received amplitude which in turn is used as an estimate of the channel phase $[5,9]$. Let us assume that $T_{1}$ is in $T x$ and sends out the MPP, while $T_{2}$ is in $\mathrm{Rx}$ and the maximum received amplitude occurs at $\phi_{k}=\phi_{12}$. Then, from Equation 1, we will get

$$
\phi_{12}=\theta_{h 2}-\theta_{h 1}-\theta_{h}
$$

While this measure does incorporate the tag-to-tag channel, it is also affected by the exciter-to-tag channels. Now, if we switch the roles of the two tags making $T_{2}$ the Tx tag and $T_{1}$ the Rx tag and repeat the same procedure, then the maximum received amplitude at $T_{1}$ occurs at $\phi_{k}=\phi_{21}$. Then from Equation 2, we get:

$$
\phi_{21}=\theta_{h 1}-\theta_{h 2}-\theta_{h}
$$

Then from Equations 3 and 4, we get

$$
\theta_{h}=-\frac{\left(\phi_{21}+\phi_{12}\right)}{2}
$$

In this way, we can think of the channel phase measure to comprise two halves each of which is computed at one of the ends of the link utilizing MPP. Then, using basic tag-to-tag communication [8], each tag can convey its half-measure to the other. Following this, both tags can simultaneously compute the accurate channel phase. This measure represents the pure tag-to-tag channel phase unaffected by the exciter to tag channels. Figure 2 shows the sequence of communication that the two tags follow in order to compute $\theta_{h}$.

\subsection{Channel Amplitude Measurement}

To measure the channel amplitude, per the existing technique, the Tx tag transmits the MPP probes while the Rx tag examines and 


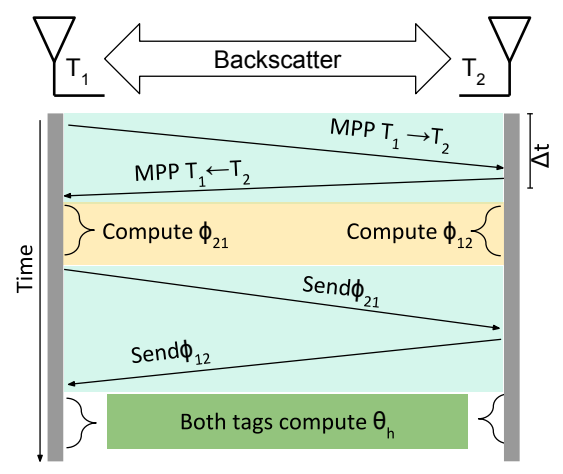

Figure 2: Channel phase calculation. The channel is assumed to remain constant during time $\Delta t$.

records the values of the maximum and minimum received amplitudes. The difference between these two is used as the channel amplitude measure. From equation 1, we get the maximum and minimum received amplitudes and the difference between them as:

$$
\begin{aligned}
\mathrm{S}_{T_{2}}^{\text {max }}= & A_{E}\left(a_{h 2}+\rho a_{h 1} a_{h}\right) ; \mathrm{S}_{T_{2}}^{\text {min }}=A_{E}\left(a_{h 2}-\rho a_{h 1} a_{h}\right) \\
& \left(\mathrm{S}_{T_{2}}^{\text {max }}-\mathrm{S}_{T_{2}}^{\text {min }}\right)=\Delta \mathrm{S}_{T_{2}}=2 A_{E} \rho a_{h 1} a_{h}
\end{aligned}
$$

In equation 6, similar to the phase measure, the channel amplitude estimated includes the exciter channel in addition to the tag-to-tag channel. This is the approach taken in prior work [5, 9]. In order to remove the effect of exciter channel effects we propose a similar technique to the phase estimation. It is based on bi-directional MPP, information exchange between two tags followed by simultaneous computation of channel amplitude.

Note that if there is no backscattering and both the tags in Figure 1 are in receive mode, then the signals that they receive will respectively be $A_{E} \cdot a_{h 1} \cdot e^{j \theta_{h 1}}$ and $A_{E} \cdot a_{h 2} \cdot e^{j \theta_{h 2}}$. We further note that both the tags employ an envelope detector for receiving, and that the exciter sends a $C W$ signal without any baseband modulation. As a result, when there is no backscattering, the amplitude of the signal at the output of the envelop detectors of the two tags will be $A_{E} \cdot a_{h 1}$ and $A_{E} \cdot a_{h 2}$ respectively. With this measurement available, $A_{E} a_{h 1}$ can then be cancelled out from the term in equation 6 to determine the pure tag-to-tag channel amplitude, $a_{h}$. Figure 3 shows the sequence of communication and operations in the two tags used to compute $a_{h}$.

Since our proposed techniques are based on bi-directional MPP followed by combined processing, we must assume that the channel is unchanged during the two (back-and-forth) MPP transmissions. Since a typical MPP cycle (about $50-100 \mu \mathrm{s}$ ) is several orders of magnitude faster than any human activity, this assumption is true for human activity sensing applications.

\section{HARDWARE IMPLEMENTATION AND EVALUATION}

\subsection{Setup}

For experimental demonstration, a prototype RF tag has been design and fabricated. This tag implements a multi-phase modulator and passive envelope detection based demodulator. The tag integrates a custom-made single dipole antenna on a separate printed circuit board (PCB). The modulator comprises an RF switch that

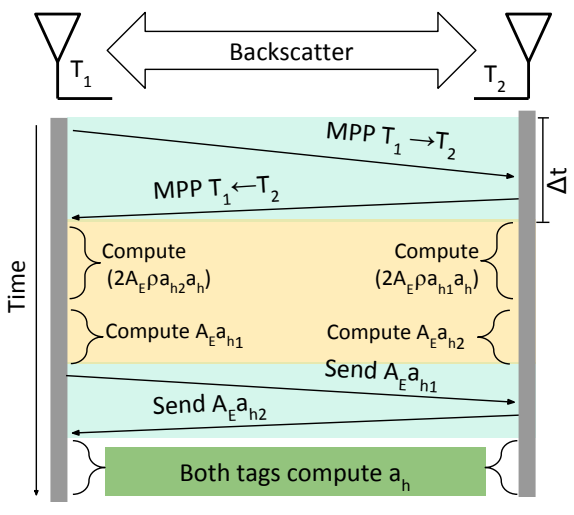

Figure 3: Channel amplitude calculation. The channel is assumed to remain constant during time $\Delta t$.

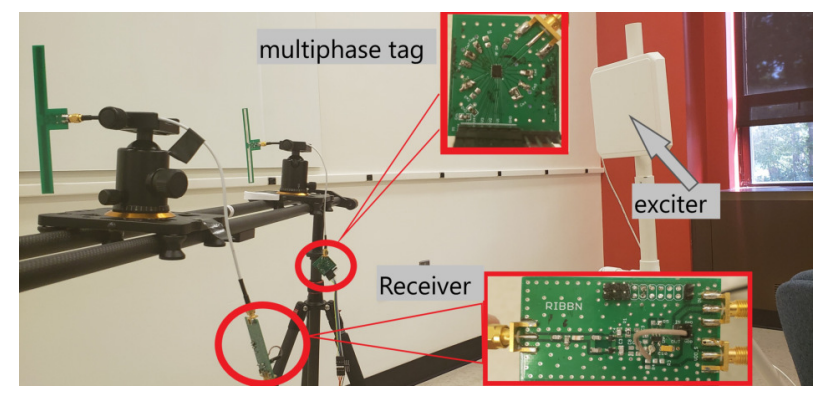

Figure 4: Experimental setup

enables selection of one of ten terminating impedances in the antenna circuit. The selection logic is implemented on a low-power microcontroller. The impedances are preselected to provide a specific reflection phase and the set of ten reflection phases uniformly covers the range from $0^{\circ}$ to $180^{\circ}$ degrees (the MPP offsets $\phi_{k}$ ). In the demodulator design, the output voltage of the envelope detector is recorded using separate PCB that integrates high-resolution 16-bit $80 \mathrm{kbps}$ analog-to-digital converter (ADC). The RF tag implementation that integrates envelope detection with on-board ADC is being designed.

Figure 4 shows a picture of the experimental setup used for validation. We use a $\mathrm{CW}$ generator at $915 \mathrm{MHz}$ connected to a circularly polarized panel antenna as the excitation source. The exciter is set to a power level of $13 \mathrm{dBm}$ at $1 \mathrm{~m}$ to $3 \mathrm{~m}$ away from the tags depending upon the experiment. During the experiments, data is collected for tag-to-tag distances of $1 \mathrm{ft}-2 \mathrm{ft}$. In each experiment, we gather data for varying channel conditions and compare the estimates obtained by our proposed techniques with those obtained by the current state-of-the art passive channel estimation methods $[5,9]$ that unlike our work here include the exciter channel in the estimation.

\subsection{Experiments and Results}

We conduct two experiments for phase measurement. In the first one, the position of exciter is changed while keeping both tags fixed. Naturally, this changes the exciter-to-tag channels $\left(\mathbf{h}_{1}\right.$ and $\mathbf{h}_{2}$ ). However since the positions of the tags are unchanged, tagto-tag channel (h) should remain constant, notwithstanding any 


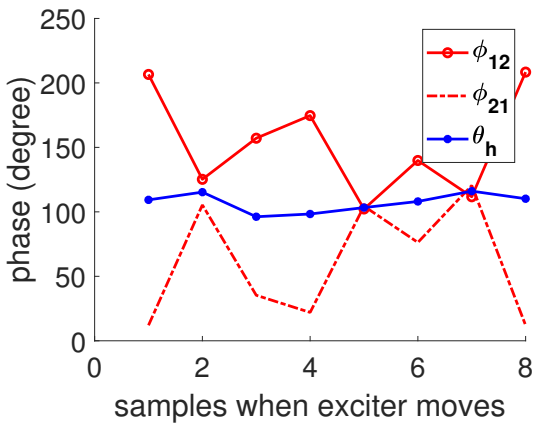

(a) phase (moving exciter)

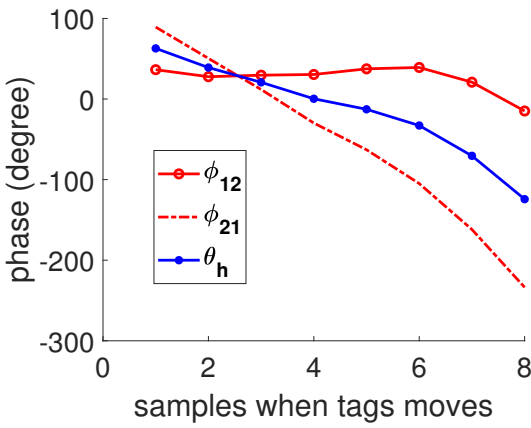

(b) phase (moving tags)

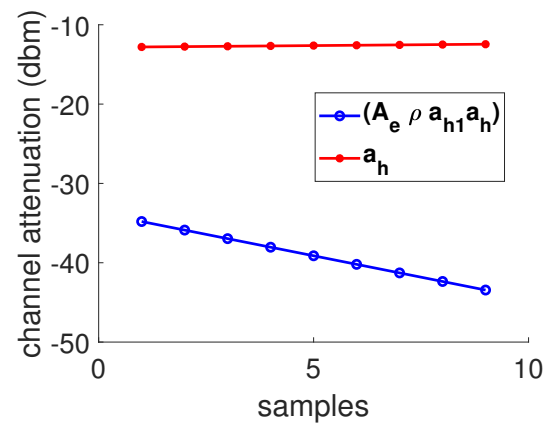

(c) amplitude

Figure 5: Experimental Results. Blue lines reflect the proposed technique and the red lines reflect prior work [5, 9].

other changes in the vicinity of the tags. For each position of the exciter, we have both tags perform MPP probing and independently compute channel phase estimate using techniques in $[5,9]$. Then, both tags collaboratively compute the phase estimate using our proposed method (Figure 2). The results are shown in Figure 5(a). The red lines show the phase estimates at the individual tags using prior methods in $[5,9]$. There, a change in the position of the exciter causes significant fluctuations in the phase estimate even though the tag-to-tag channel is unchanged. On the other hand, the phase estimate using our technique (blue line) is almost constant. This clearly shows the validity of our technique in isolating or filtering out the effects of changes in the exciter to tag channels from the tag-to-tag channel phase measurement.

In the second experiment, the exciter is kept fixed while both tags are moved apart along a single line. We see, in Figure 5(b), that the channel phase measurement using proposed technique correspond to what is theoretically expected, i.e. linear variation.

The phase measurements using the proposed method could have a wrapping effect. Any machine learning model using the change in phase for gesture recognition can use continuity principle to unwrap it. All graphs in this section have been corrected for it.

Finally, we conduct an experiment to compare the tag to tag channel amplitude measure as previously reported (i.e., $A_{E} \rho a_{h 1} a_{h}$ ) and our proposed pure channel amplitude (i.e., $a_{h}$, see Section 2.3). During the experiments the tags are moved closer to each other in a way that $a_{h}$ should increase. Simultaneously the exciter to Tx channel is changed such that the $A_{E} a_{h 1}$ is drastically decreased. We see in Figure $5 c$ that the channel amplitude measure per previously reported techniques (as in $[5,9]$ that use equation 6 directly) goes down drastically (blue) even though the tags are actually moving closer.

On the other hand, our proposed channel amplitude measure is slightly increasing (red). Hence, as in the case of phase estimation, our method effectively isolates the exciter to tag channel from the tag to tag channel.

\section{CONCLUSION}

Recent advances in Backscattering Tag to Tag Networking (BTTN) has enabled its use in human activity sensing by developing methods for passive tag-to-tag channel estimation. We proposed and implemented collaborative passive channel estimation in BTTN which overcomes the limitations of prior passive channel estimation techniques. Our methods enable estimation of the pure tag-to-tag channel without including any effects of the exciter channel. This improves the effectiveness, including better localization, classification and sensing multiple activities in the vicinity at the same time using different tags. While for simplicity we have not yet performed actual human activity sensing in the reported experiments, we have demonstrated the underlying channel estimation techniques and shown their superiority in overcoming the limitations of the state-of-the-art.

\section{ACKNOWLEDGEMENT}

This work is supported by NSF grant CNS-1763843.

\section{REFERENCES}

[1] Dinesh Bharadia, Kiran Raj Joshi, Manikanta Kotaru, and Sachin Katti. 2015. Backfi: High throughput wifi backscatter. ACM SIGCOMM Computer Communication Review 45, 4 (2015), 283-296.

[2] Daniel M Dobkin. 2012. The RF in RFID: UHF RFID in Practice. Newnes.

[3] Joshua F Ensworth and Matthew S Reynolds. 2015. Every smart phone is a backscatter reader: Modulated backscatter compatibility with bluetooth 4.0 low energy (BLE) devices. In 2015 IEEE International Conference on RFID (RFID). IEEE, $78-85$.

[4] Kiran Joshi, Dinesh Bharadia, Manikanta Kotaru, and Sachin Katti. 2015. WiDeo: Fine-grained Device-free Motion Tracing using RF Backscatter. In 12th USENIX Symposium on Networked Systems Design and Implementation (NSDI 15). 189-204.

[5] Y. Karimi, Y. Huang, A. Athalye, S. Das, P. Djurić, and M. Stanaćević. 2019. Passive Wireless Channel Estimation in RF Tag Network. In 2019 IEEE International Symposium on Circuits and Systems (ISCAS). 1-5.

[6] Vincent Liu, Aaron Parks, Vamsi Talla, Shyamnath Gollakota, David Wetherall, and Joshua R Smith. 2013. Ambient backscatter: wireless communication out of thin air. In ACM SIGCOMM Computer Communication Review, Vol. 43. ACM, 39-50.

[7] Aaron N Parks, Angli Liu, Shyamnath Gollakota, and Joshua R Smith. 2014. Turbocharging ambient backscatter communication. In ACM SIGCOMM Computer Communication Review, Vol. 44. ACM, 619-630.

[8] Jihoon Ryoo, Jinghui Jian, Akshay Athalye, Samir R Das, and Milutin Stanaćević. 2018. Design and Evaluation of BTTN: A Backscattering Tag-to-Tag Network. IEEE Internet of Things fournal 5, 4 (2018), 2844-2855.

[9] Jihoon Ryoo, Yasha Karimi, Akshay Athalye, Milutin Stanaćević, Samir R Das, and Petar Djurić. 2018. BARNET: Towards activity recognition using passive backscattering tag-to-tag network. In Proceedings of the 16th Annual International Conference on Mobile Systems, Applications, and Services. ACM, 414-427.

[10] Nguyen Van Huynh, Dinh Thai Hoang, Xiao Lu, Dusit Niyato, Ping Wang, and Dong In Kim. 2018. Ambient backscatter communications: A contemporary survey. IEEE Communications Surveys \& Tutorials 20, 4 (2018), 2889-2922.

[11] C. Xu, L. Yang, and P. Zhang. 2018. Practical Backscatter Communication Systems for Battery-Free Internet of Things: A Tutorial and Survey of Recent Research. IEEE Signal Processing Magazine 35, 5 (Sep. 2018), 16-27.

[12] Pengyu Zhang, Dinesh Bharadia, Kiran Joshi, and Sachin Katti. 2016. Hitchhike: Practical backscatter using commodity WiFi. In Proc. ACM Sensys. 259-271. 